\title{
Analysis of the isotropic models of the Mullins effect based on filled silicone rubber experimental results
}

\author{
G. Machado, G. Chagnon*, D. Favier \\ Université de Grenoble/CNRS, Laboratoire 3S-R, Cedex 9, 38041 Grenoble, France.
}

\begin{abstract}
The Mullins effect of rubber like material is classically defined as the stress softening during initial loading cycles. This effect is not accounted when the mechanical properties of material are modeled by a simple hyperelastic strain-energy function. In order to capture the stress softening it is necessary to define a set of supplementary variables as well a dissipation function, which evolves with the deformation history. In this paper we first describe experimental results that illustrate stress softening in particle-reinforced silicone rubber for uniaxial, planar and equibiaxial traction. The results allow to analyze the stress softening for the three different load cases. First, with respect to the choice of a stress-softening measure, the energy loss was evaluated by comparing the stored elastic energy for the first and the second loadings. The results point out that the virgin energy and the first invariant parameters are the best choice. Nevertheless, the maximum principal elongation, classically used in Mullins effect modeling, is not able to describe the different load cases. Furthermore, the ability of different class of models to describe filled silicone rubber was studied. The results show that models with a non-proportional and non-homothetical second load paths seem to be more efficient.
\end{abstract}

Key words: filled silicone rubber; Mullins effect; stress-softening; deformation criteria; hyperelasticity;

\section{Introduction}

Like many filled rubber-like materials, as well as soft biological tissues and other biomaterials, filled silicone rubber exhibit highly non-linear mechanical behavior. This non-linearity is commonly characterized by large strain levels under static conditions, a nonlinear stress-strain response and strain rate dependence. Finally, under cyclic loading conditions, hysteresis and stress-softening behaviors are observed on both filled and unfilled elastomers. For the last half century all these cyclic phenomena were evidenced and modelled to support the most widely engineering applications. However, their microscopic mechanisms (breaking of weak chains, breaking of links, desabsorption of chains, etc.) explanation remains non-unanimous.

Hysteresis is rather related to the dissipative nature of material, i.e., related with viscoelasticity (Bergstrom and Boyce, 2000) and viscoplasticity (Miehe and Keck, 2000) behaviors. It is characterized as the difference between the loading and unloading paths during a cycle. On the other hand the stress-softening phenomenon, also called Mullins effect (Mullins, 1969), can be described as a stress-softening phenomenon of the material after a first loading. It can be idealized as an instantaneous and irreversible softening of the stress-strain curve, due to rearrangements in the microstructure of the material that occurs whenever the load increases beyond its prior all-time maximum value. At times when the load is less than a prior maximum, nonlinear elastic behavior prevails. One time the previous maximum stretch is reached the loading path turns up and follows the primary curve again up to a new maximum.

\footnotetext{
* Corresponding author

Email address: gregory.chagnon@grenoble-inp.fr (G. Chagnon)
} 
Through the years, the Mullins effect has experimentally been observed in different deformation states and numerous models have been proposed, but there is non-unanimous opinion on the choice of the variable to describe the evolution of the Mullins effect. Many different criteria have been chosen during the last fifty years, the most used remains the strain energy density or the maximum principal elongation. Within this context, under the assumption of isotropy, the aims of this work is to analyze which are the most suitable modelling for Mullins effect for different loading cases. In this way, a filled silicone rubber is studied under various mechanical loadings, where the Mullins effect becomes evident. Then, the determination of the best criterion to describe the evolution of stress softening and the kind of model able to describe it for every loading cases are evaluated. Hence, the different type of modelling are summarized in 2. Material preparation procedures are described in Section 3, testing procedures and strain field measurements techniques based on optical methods are described in Section 4. Experimental results are then presented, in Section 5. An analyze of the strain measure criteria and the best way to model Mullins effect is finally presented in Section 6.

\section{Mullins effect modelling}

There exist a large number of Mullins effect constitutive equations, with different kind of isotropic or anisotropic modelling hypothesis. More and more modelling tackles the anisotropy of the Mullins effect (see for example Ehret and Itskov (2009), Diani et al. (2006) and Miehe and Göktepe (2005)). In this paper, we focus on isotropic approaches of the Mullins effect to analyze what are the best ways of modelling. Whatever is the modelling, a deformation measure parameter is used to control Mullins effect evolution and its choice is critical.

\subsection{Choice of the modelling}

A classical approach to describe Mullins effect is to use damage mechanic theory. The strain energy density of a virgin material $\mathcal{W}_{0}(\mathbf{F})$ submitted to the deformation gradient $\mathbf{F}$ can be modified incorporating continuum damage parameter $d$ in order to take into account the stress softening. The classical form is given by

$$
\mathcal{W}(\mathbf{F})=(1-d) \mathcal{W}_{0}(\mathbf{F})
$$

All proposed models vary from each other by the definition of the damage criteria and the form of the damage evolution function. The definition can involve any microstructural chain damage and microvoid formations both associated with physical phenomena like breaking of chains and/or links, desabsorption of chains, etc. A common approach assumes that damage is a function of a discontinuous quantity like the maximum applied stretch or the maximal energy density with respect to the primary load path. Nevertheless, as reported by Chagnon et al. (2004), these models assume that the stress ratio between two different secondary load curves are related by a constant multiplicative factor:

$$
\frac{P_{s}^{j}}{P_{s}^{i}}=\frac{1-d\left(x_{s}^{j}\right)}{1-d\left(x_{s}^{i}\right)}
$$

where $s$ denotes the secondary load curves numbered $i=1, \ldots, 3$ and $j=2, \ldots, 4(i<j)$ for the presented experimental data.

Pseudo elasticity models are based on a different approach. Ogden and Roxburgh (1999) present an additive function to describe the second loading curves, this permits to have two distinguished functions to describe the first and the second loading curves. In this case some conditions must be imposed to ensure the continuity of the strain-stress behavior. Now, no more proportionality is imposed between the second loading curves and the evolution function for the second loading curves is described thanks to $\mathcal{W}_{0}$ measure. This permits to have a model able to characterize the different loading cases. This approach has been very employed in the past few years (see for example Dorfmann and Ogden (2004) and Horgan et al. (2004)).

The double network theory is different from damage mechanics as the multiplicative function used for describing Mullins effect is not constant on the second loading curves. It can no longer be considered as a 
Table 1: Definition of damage criteria for some representative models

\begin{tabular}{|c|c|c|}
\hline Variable & Description & Reference \\
\hline$\sqrt{2 \mathcal{W}_{0}(\mathbf{F})}$ & & $\begin{array}{l}\text { Simo (1987), Miehe and Keck (2000), } \\
\text { Septanika and Ernst (1998) }\end{array}$ \\
\hline $\mathcal{W}_{0}(\mathbf{F})$ & & $\begin{array}{l}\text { Ogden and Roxburgh (1999), } \\
\text { Laraba-Abbes et al. (2003), Miehe (1995) }\end{array}$ \\
\hline$\lambda_{\max }$ & Maximum largest principal stretch & Laiarinandrasana et al. (2003) \\
\hline$\sqrt{I_{1} / 3}-1$ & $I_{1}=\operatorname{trace}(\mathbf{C})=\operatorname{trace}(\mathbf{B})$ & $\begin{array}{l}\text { Chagnon et al. (2004), } \\
\text { Qi and Boyce (2004) }\end{array}$ \\
\hline$\varepsilon: \varepsilon$ & $\varepsilon=\frac{1}{2}\left(\mathbf{C}^{-1}-\mathbf{I}\right)$ & Lion (1996) \\
\hline$\sqrt{\mathbf{B}: \mathbf{B}}$ & $\mathbf{B}=\mathbf{F F}^{T}$ & Zuñiga and Beatty (2002) \\
\hline$\sqrt{\mathbf{e}: \mathbf{e}}$ & $\mathbf{e}=\frac{1}{2}\left(\mathbf{I}-\mathbf{B}^{-1}\right)$ & Krishnaswamy and Beatty (2000) \\
\hline$P_{\max }$ & Maximal principal stress & Johnson and Beatty (1993) \\
\hline
\end{tabular}

damage function as the value of the function is evolving on second loading curves and it comes back to a value of one on the first loading curve whereas the material has already be stretched. DeSouza Neto et al. (1994), based on the idea of Gurtin and Francis (1981) proposed a damage master curve, that describes the evolution of the network, that is magnified to the considered maximum previous strain level.

A physical approach, proposed by Marckmann et al. (2002) is based on the position of the strain hardening when the material reaches it maximum previous deformation level. They assume that the macro-molecular chain network is evolving with the maximum deformation, they assume that the number of active monomers is constant. This hypothesis was relaxed by Chagnon et al. (2006) who considered that some chains can become hanging, that means that the number of active monomers is a decreasing function of the deformation. In another way, Qi and Boyce (2004) developed a double network model also based on evolution of chain elongation deformation measure. This permit to have independent second loading curves.

As shown, there exists a large kind of models (all existent models have not been presented here) but it seems that the choice of an energy deformation measure is quite important. This choice ensures an ability of the model to describe the different load cases, moreover the different modelling have different consequences on the strain-stress curves.

\subsection{The history parameter choice}

The choice of the Mullins effect evolution criterion is fundamental for the description of different loading cases. As pointed out by Diani et al. (2009), many criteria are exhibited by different authors. The Table 1 summarizes different choices of the past years. Usually, the choice of the measure is not justified, but is able to represent the experimental curves. For an uniaxial representation, the choice of the parameter is not essential, as all can describe the stress softening. The Mullins effect evolution function is then built in function of this parameter. Nevertheless, in a tridimensional representation, the choice becomes very crucial as it rules the position of the second loading curves in the different deformation modes. In the next sections the experimental procedures and results are provided to enhance this discussion. 


\section{Preparation of the Silicone Specimens}

The samples used in the experiments were made of a filled silicone rubber called Rhodorsil RTV3428 supplied as two liquid components: the uncured silicone and the curing agent. The final material is produced by a polyaddition, curing at room temperature. The liquid mixture is molded by injection to obtain a sheet with constant thickness. Although the experiments are performed separately and the strain states are different, data from all of the individual experiments is often used as a set. This means that the specimens used for each of the experiments must be made using the same protocol of elaboration, in order to obtain specimens with reproducible mechanical properties, as follow: (i) mixing the components with a 10/1 mass ratio, (ii) putting the mixture under vacuum for 20 minutes in order to eliminate undesirable entrapped air bubbles, (iii) sheet molding injection, (iv) putting mold inside an oven at $70^{\circ} \mathrm{C}$ for 4 hours in order to accelerate the curing process and assure a sufficient cross-linking density; (v) sheet demolding after 1 hour exposed at ambient temperature. Finally, the external surface of the molded plate was coated with a stochastic silicone paint pattern (Meunier et al., 2008). Made of small speckles, the pattern is necessary for the digital image correlation (DIC) and stereo digital image correlation (SDIC) field measurements. Note that the quality of the coated pattern (size, density and grey contrast level) is a critical point to obtain a good estimation of the strain field measurement.

\section{Testing procedures and strain field measurements techniques}

\subsection{In-plane tests}

In plane quasi-static experiments were conducted on a MTS 4M universal testing machine with an Entran ELPM-T2 $2250 \mathrm{~N}$ load cell. The images were recorded at $0.5 \mathrm{~Hz}$ with a Jai TM-4200GE CCD camera using a reduced scan of $2048 \times 1000$ pixels. Figure 1 presents the experimental setup. First, simple tensile tests

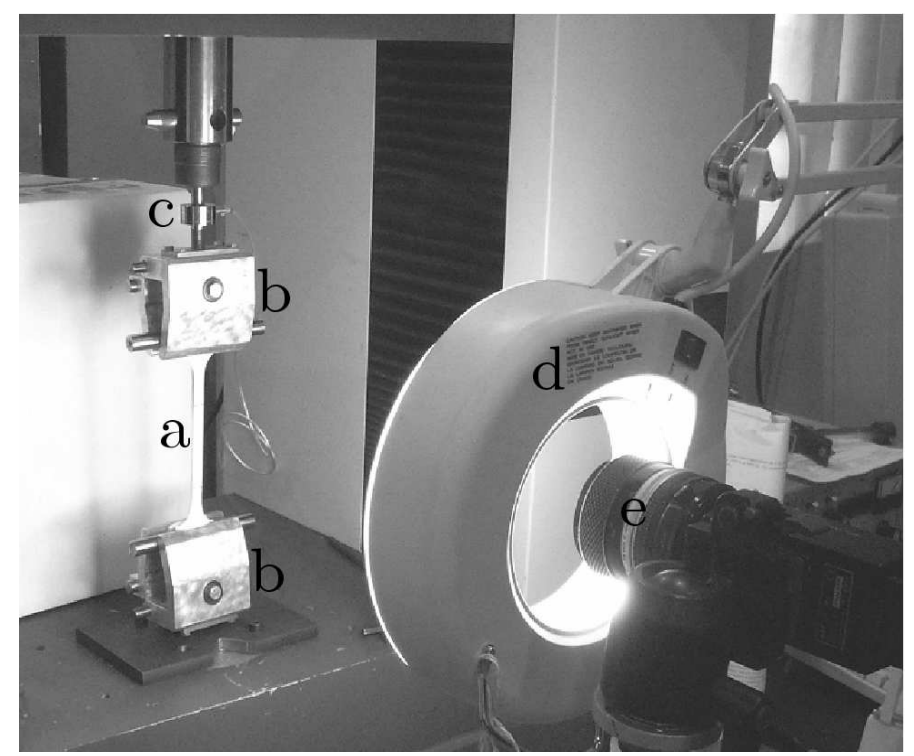

Figure 1: In-plane experimental setup. (a) silicone specimen; (b) clamps; (c) load cell; (d) omni light; and (e) CCD camera.

were performed on rectangular samples having an initial gauge length $l_{0}=40 \mathrm{~mm}$, width $w_{0}=13 \mathrm{~mm}$ and a thickness $e_{0}=2 \mathrm{~mm}$. Since the experiment was not intended to fail the specimen, there was no need to use a dumbbell shaped specimen commonly used to prevent specimen failure nearby the clamps. But knowing that the effects of clamps create an indeterminate state of stress and strain in the region surrounding the clamp, due to the process of gripping, the initial gauge length was adopted as being less than the real 

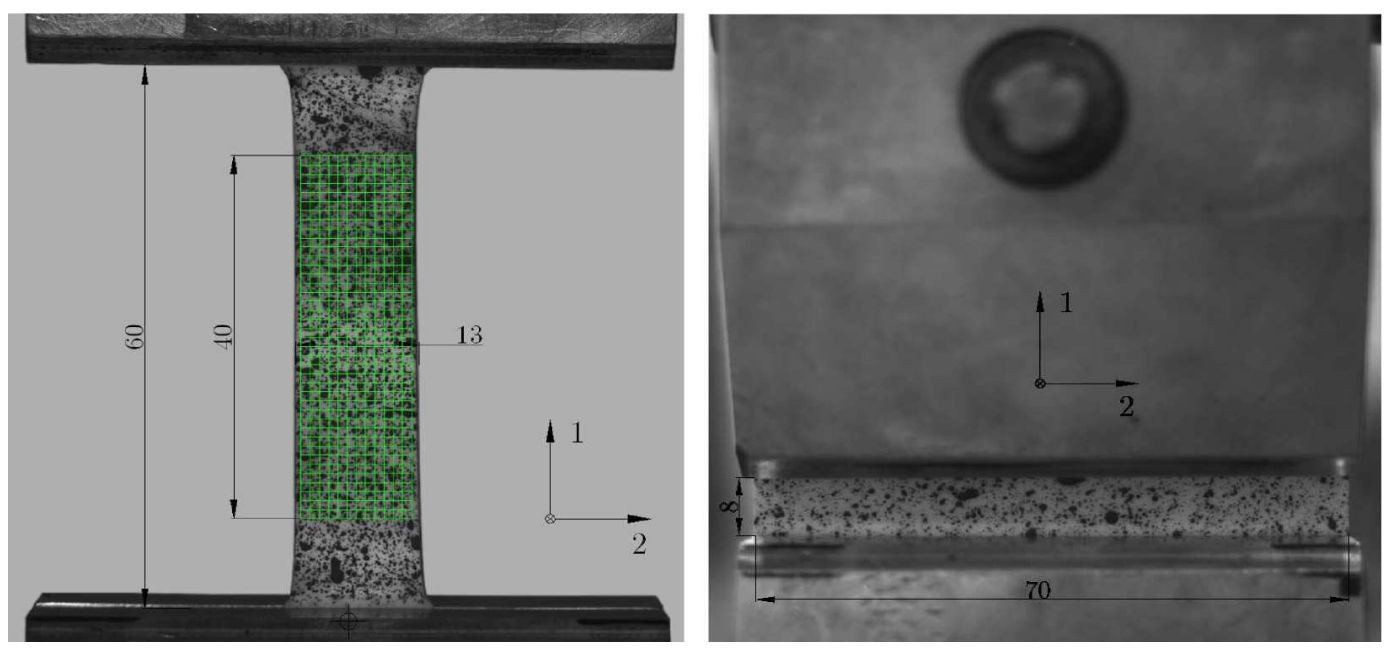

Figure 2: Test specimen dimensions (a) uniaxial tensile specimen (b) planar tensile specimen.

physical size of the samples. Figure 2(a) presents the tensile test specimen. Second, the pure shear strain state was approached by performing planar shear tensile test (see Figure $2(\mathrm{~b})$ ). The initial height $l_{0}$, the constant width $w_{0}$ and the thickness $e_{0}$ of samples were $8 \mathrm{~mm}, 70 \mathrm{~mm}$ and $2 \mathrm{~mm}$, respectively. These dimensions have as objective to create an experiment where the specimen is perfectly constrained in the lateral direction such that all specimen thinning occurs in the thickness direction.

The DIC technique, like a non-contact method, is often used to characterize rubber-like materials, see for example Meunier et al. (2008) and Sasso et al. (2008). In each testing time step an image of the deformation of samples was recorded using a CCD camera. Using the DIC, it is possible to reconstruct the surface of the deformed samples and determinate the full-field surface displacements. An accuracy on the order of $10^{-2}$ pixels or better for in-plane displacement components and point-to-point strain accuracy of $\pm 10^{-4}$ in-plane can be obtained. The DIC method is preferred because there is no change in stiffness resulting from the presence of a attached sensor (classical extensiometry) and for being insensitive to ambient vibrations and rigid body motions (speckle pattern interferometry). Moreover the method is able to deal with high strain levels, what is a very desirable feature given the high strain level experienced by the tested material. Also the DIC allows demonstrating and measuring an heterogeneous deformation field. See Sutton (2008) for further explanation about digital image correlation method.

\subsection{Out-plane tests}

A bulge test was also conducted in order to determinate a equibiaxial state. This test, also called "balloon" test, consists of a thin disk specimen, of initial diameter $d_{0}=200 \mathrm{~mm}$ with $e_{0}=2 \mathrm{~mm}$ of thickness, constrained between two clamping flanges. This test machine, performed at the same way in Meunier et al. (2008), consists of a air piston connected to the bottom circumferential clamp. The internal pressure is measured with a pressure sensor Foxboro 0-500 mbar. For these tests, out-of-plane displacements measurements were needed, therefore a Dantec Q-400 Stereo Digital Image Correlation (SDIC) system was used to acquire and correlate the images. Figure 3 presents the experimental setup. Since a large out plane displacement is experienced by the silicone sample, a good focal distance is absolutely necessary. Nevertheless, a high spatial resolution is needed to permit an accurate measurement. Using the stereocorrelation it is possible to reconstruct the deformed sample surface geometry as well as determinate the displacement and strain fields. Because rigid body motion has no effect on the measurements, a high level of strain has been achieved in this kind of experiment. The Figure 4 shows the strain level curves for the maximal pressure value of $18 \mathrm{KPa}$ in the center of the membrane. The curvature on the top of the bulge is computed, using a post-treatment routine. 


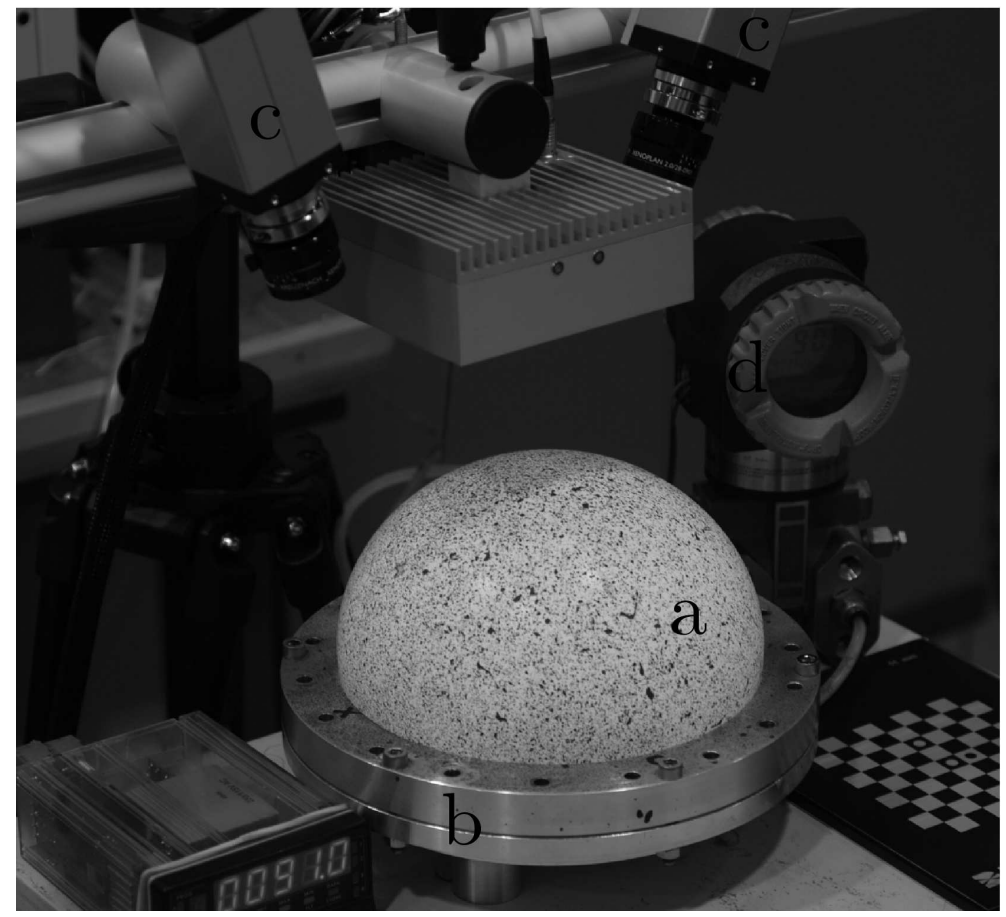

Figure 3: Bulging test set-up. (a) bulge specimen; (b) circular clamp; (c) CCD cameras; and (d) pressure sensor.

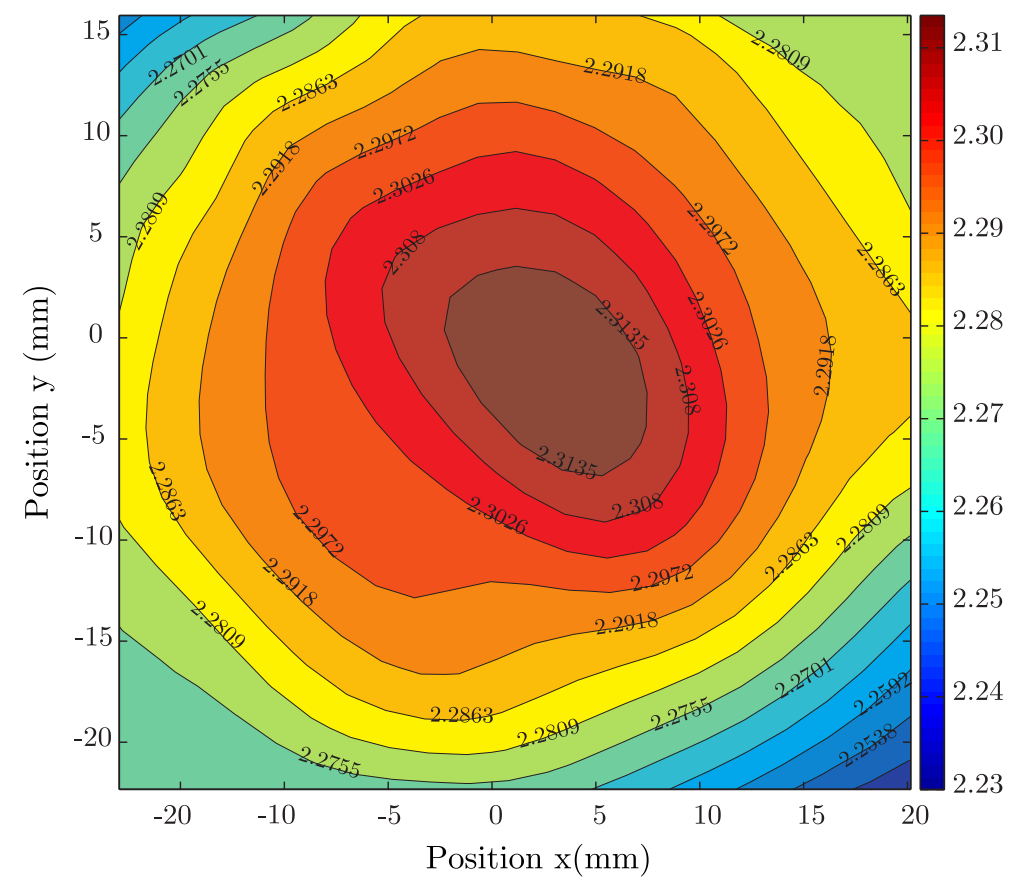

Figure 4: First principal strain (Green-Lagrange measure) in the center of the membrane for the maximal pressure value of 18 $\mathrm{KPa}$. 


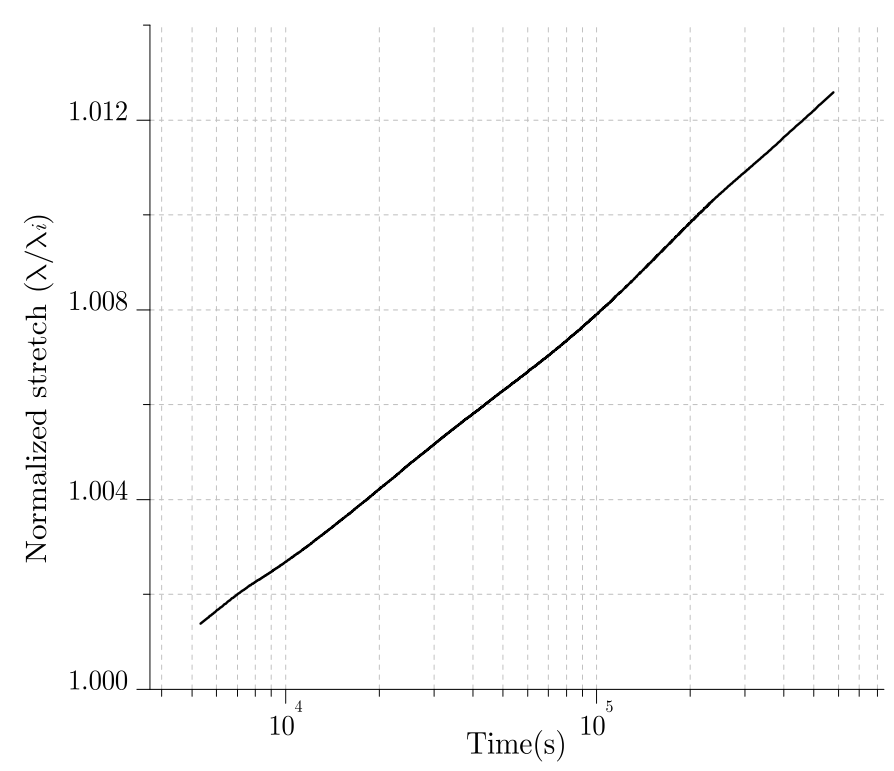

Figure 5: Time evolution of the normalized stretch for a creep test performed at constant load $20 \mathrm{~N}$ recorded after a monotonic tensile resulting in $\lambda_{i}=2.160$.

\section{Experimental results}

Different tests are realized to evaluate the Mullins effect in different loading cases. First, time dependence of the material is studied and then the stress-softening is analyzed.

\subsection{Time dependent response}

A creep tensile test was performed, where a constant monotonic load of $20 N$ was applied resulting in a initial stretch of $\lambda_{i}=2.160$. It can be observed in Figure 5 that the material response tends towards an equilibrium state, which cannot be reached within laboratory time scale. However the stretch increased by less than $1 \%$ within the observed time range. In a second time, a tensile specimen has been subjected to a load/unload sequence at different elongation rates $\dot{\lambda}=2.5 \times 10^{-3} \mathrm{~s}^{-1}$ to $1.25 \mathrm{~s}^{-1}$ in order to verify the rate-dependence influence. No noticeable difference between stress-strain responses was observed at the considered strain rate range. Consequently the RTV3428 behavior can be assumed independent of the rate of deformation for the observed ranges.

\subsection{Uniaxial tension test}

During the test, using an elongation rate of $\dot{\lambda}=1.60 \times 10^{-2} \mathrm{~s}^{-1}$, the nominal stress tensor $\mathbf{P}$ (First PiolaKirchhoff stress tensor) is assumed to be homogeneous within the gauge region as well as the deformation gradient tensor $\mathbf{F}$. Since the current thickness is not measured, the material is assumed to be incompressible, i.e., $\operatorname{det}(\mathbf{F})=1$ for convenience. In the central zone, the deformation gradient, considering that the direction 1 is the loading one, is given by:

$$
\mathbf{F}=\lambda\left(\mathbf{e}_{1} \otimes \mathbf{E}_{1}\right)+\lambda^{-1 / 2}\left(\mathbf{e}_{2} \otimes \mathbf{E}_{2}+\mathbf{e}_{3} \otimes \mathbf{E}_{3}\right)
$$

and the nominal stress tensor is:

$$
\mathbf{P}=P_{11}\left(\mathbf{e}_{1} \otimes \mathbf{E}_{1}\right)
$$

where $\left(\mathbf{E}_{\mathbf{1}}, \mathbf{E}_{\mathbf{2}}, \mathbf{E}_{\mathbf{3}}\right)$ and $\left(\mathbf{e}_{\mathbf{1}}, \mathbf{e}_{\mathbf{2}}, \mathbf{e}_{\mathbf{3}}\right)$ are the initial and deformed orthonormal basis respectively, here identical. 


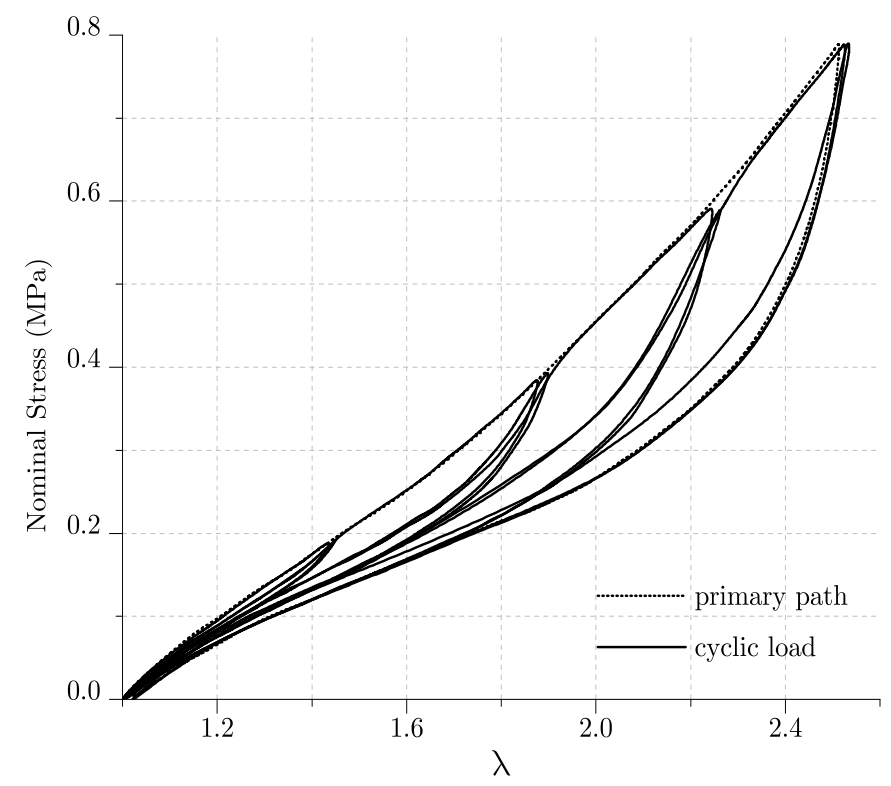

Figure 6: Cyclic loading-unloading tensile test with increasing maximum stress: $0.2,0.4,0.6$ and $0.8 \mathrm{MPa}$ at $\dot{\lambda}=1.60 \times 10^{-2} s^{-1}$.

A cyclic loading test was realized, the results are presented in Figure 6. Different phenomena are highlighted, first a large Mullins effect appears by comparing the two first loading at each strain level, but with very few residual elongation. Moreover, a little hysteresis after the first cycle (difference between loading and unloading during the second cycle) is observed.

\subsection{Plane strain tensile test}

Also, the strain state during the test was supposed to be homogeneous, by assuming the direction 1 is the loading one and direction 2 is the breadth of the specimen direction, the deformation gradient tensor was expressed as:

$$
\mathbf{F}=\lambda\left(\mathbf{e}_{1} \otimes \mathbf{E}_{1}\right)+\left(\mathbf{e}_{2} \otimes \mathbf{E}_{2}\right)+\lambda^{-1}\left(\mathbf{e}_{3} \otimes \mathbf{E}_{3}\right)
$$

where the stress tensor is

$$
\mathbf{P}=P_{11}\left(\mathbf{e}_{1} \otimes \mathbf{E}_{1}\right)+P_{22}\left(\mathbf{e}_{2} \otimes \mathbf{E}_{2}\right)
$$

A cyclic planar loading test was realized, the results are presented in Figure 7. Planar tensile response, likewise uniaxial traction, presents the same phenomena. Here, the residual stretch shows to be more evident. The maximum principal stretches experienced by the planar specimens are smaller if compared with uniaxial traction specimens. This limitation lies in the fact that the planar traction specimens must to be constrained in the lateral direction without slipping.

\subsection{Equibiaxial tension state approached by the bulge test}

Due to the axial-symmetry of the experimental configuration the equibiaxiality of the stress and strain is obtained on the top the inflated sample. The deformation gradient tensor in a local coordinate system is

$$
\mathbf{F}=\lambda_{t}\left(\mathbf{e}_{1} \otimes \mathbf{E}_{1}+\mathbf{e}_{2} \otimes \mathbf{E}_{2}\right)+\lambda_{t}^{-2}\left(\mathbf{e}_{3} \otimes \mathbf{E}_{3}\right)
$$

where $\lambda_{t}$ is the elongation in the tangential direction of the specimen measured by SDIC. Finally, with all assumptions above, the stress tensor is given by

$$
\mathbf{P}=P_{11}\left(\mathbf{e}_{1} \otimes \mathbf{E}_{1}+\mathbf{e}_{2} \otimes \mathbf{E}_{2}\right)+P_{33}\left(\mathbf{e}_{3} \otimes \mathbf{E}_{3}\right)
$$




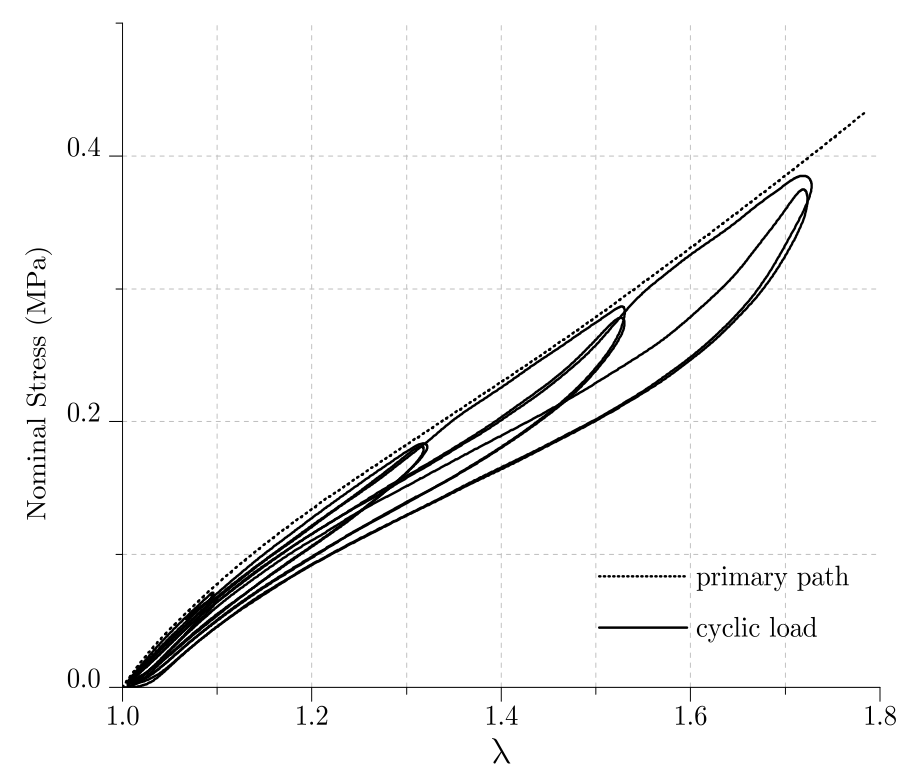

Figure 7: Cyclic loading-unloading planar tensile test. Maximum stress: $0.18,0.28$, and $0.38 \mathrm{MPa}$ at $\dot{\lambda}=2.60 \times 10^{-2} \mathrm{~s}^{-1}$.

Once thickness dimension is much smaller than the other two planar dimensions $\left(d_{0} \gg e_{0}\right)$, the stress is also assumed to be negligible along the thickness. Another consideration comes from the fact that the curvature of the inflated sample was presumed to be the same along all directions at the disk center.The component $P_{11}$ can be calculated from knowledge of curvature radius $r$, the tangential elongation $\lambda_{t}$ and the pressure $p$ recorded during the test:

$$
P_{11}=P_{22}=\frac{p r}{2 e_{0}} \lambda_{t} ; P_{33} \approx 0
$$

The results for the central area are presented in Figure 8 for a cyclic loading. The response are qualitatively similar to uniaxial loadings with hysteresis and Mullins effect.

\section{Discussion}

On the one hand, by use of experimental data, we propose to analyze the consequence of the choice of a stress-softening measure. Like, often described, by considering that the main part of the Mullins effect is represented by the difference between the first and the second loading paths, the energy loss is evaluated by comparing the stored elastic energy for the first and the second loadings. The evolution of the ratio of these two quantities is plotted according to all the Mullins effect measure parameters of Table 1 , for the three loading cases: uniaxial and equibiaxial extensions and planar tension. The results are presented in Figure 9. It can be noticed that none of the parameters present a perfect correlation for all loading cases. Although, for the tested strain range, it seems that the virgin energy $\left(\mathcal{W}_{0}\right)$ and the first invariant $\left(I_{1}\right)$ parameters are the best choice. The maximal principal stress $\left(P_{\max }\right)$ can be acceptable for low strains levels. What clearly appears is that the maximum principal elongation $\left(\lambda_{\max }\right)$, usually used in Mullins effect modelling, is not able to describe the different loading cases. All the other parameters derived from the trace operator of a strain measure are not more efficient. All these measures become less and less efficient at large deformation. It emphasizes the fact that the Mullins effect should be described by $\mathcal{W}_{0}$ or $I_{1}$ parameter. All this means that the use of a non-efficient parameter implies the construction of the second loading modelling on wrong bases. Whatever is the model, the evolution function used to describe the stress-softening will be fitted on 


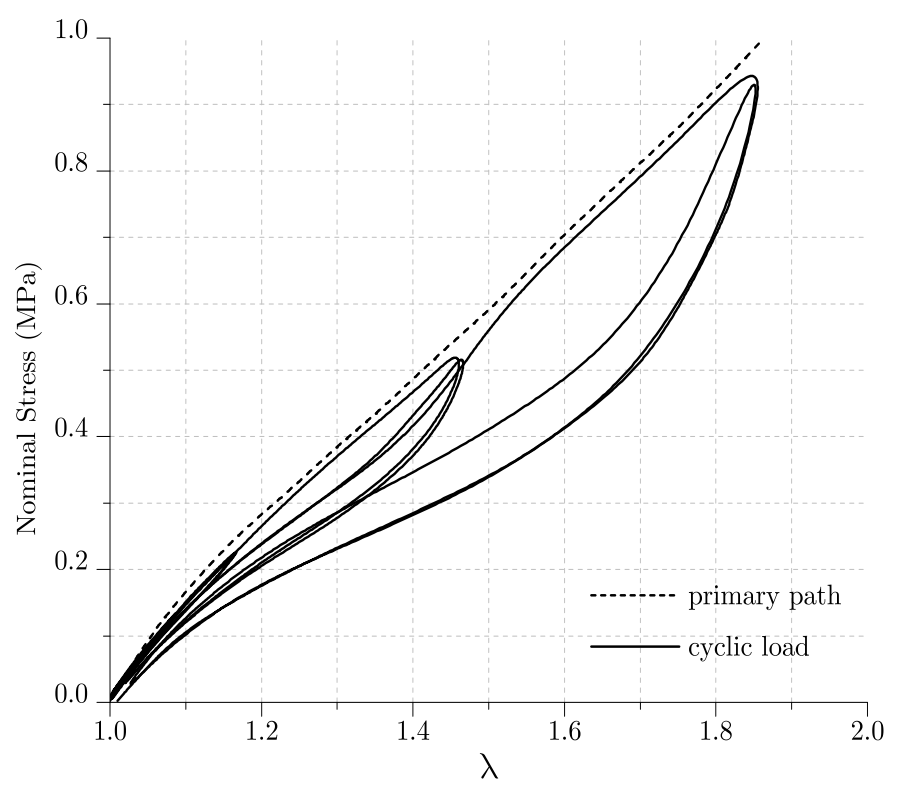

Figure 8: Cyclic equibiaxial stress-strain behavior obtained using the tension bulge test.

a non-adequate parameter. Many Mullins effect modelling have a large number of parameters, this permits to have a good fit, but if the wrong deformation criterion is used, the physics of the phenomenon is lost.

On the other hand, some modelling hypothesis are discussed. A damage modelling approach imposes to these ratios to be constant whatever is the maximum deformation parameter. Figure 10 presents the ratios between the different second loading curves (plotted according to the principal elongation). The beginning of the curves varied very quickly, but this is due to experimental errors which are more significative at low strain levels. It clearly appears that no damage model can correctly represent the Mullins effect of a material that has a similar behavior than the RTV3428 silicone. A choice must be done, but the beginning of second loading curves and strain hardening cannot be described correctly at the same time using these models. The proportionality between the second loading curves prevents from describing large strain hardening, then it can only be applied to material that have a weak influence of Mullins effect. But this discards the imposition of proportionality between second loading curves, like proposed by Beatty and Krishnaswamy (2000) and Zuñiga and Beatty (2002). But their proposition is written thanks to a strain measure that does not seem to be very efficient to characterize all loading cases. This was improved by Zuñiga (2005) by changing the strain measure to an energy measure. An interesting analysis and criticism of this model is proposed by Kazakeviciute-Makovska (2007).

DeSouza Neto et al. (1994) proposed a master second loading curve to describe Mullins effect, the curve is magnified to join the stress-free state to the maximum deformation. Figure 11 presents a normalization of the strain-stress curve for the three simple tests for the maximum strain and the normalized strain energy. It clearly appears that all the curves are not superimposed, that means that the idea of a single master curve is not adequate. The form of the second loading curve is varying a lot, according to the maximum deformation. The strain hardening is very different and is not appearing at the same in a second loading curve. This phenomenon is also more important for Natural Rubber because of strain induced crystallization.

All these remarks emphasize the fact that an efficient Mullins effect modelling, by an isotropic approach, must not use a formalism where the second loading curves are proportional or homothetical. The modelling should be able to describe different form of strain-hardening according to the maximum previous deformation level. Moreover governing Mullins effect parameter should be built thanks to different loading cases; a simple uniaxial test can drive to abnormal conclusion. We suggest to use the elastic energy or the first strain invariant. The physical or pseudo-elastic approaches seems to be the best way to tackle correctly Mullins 

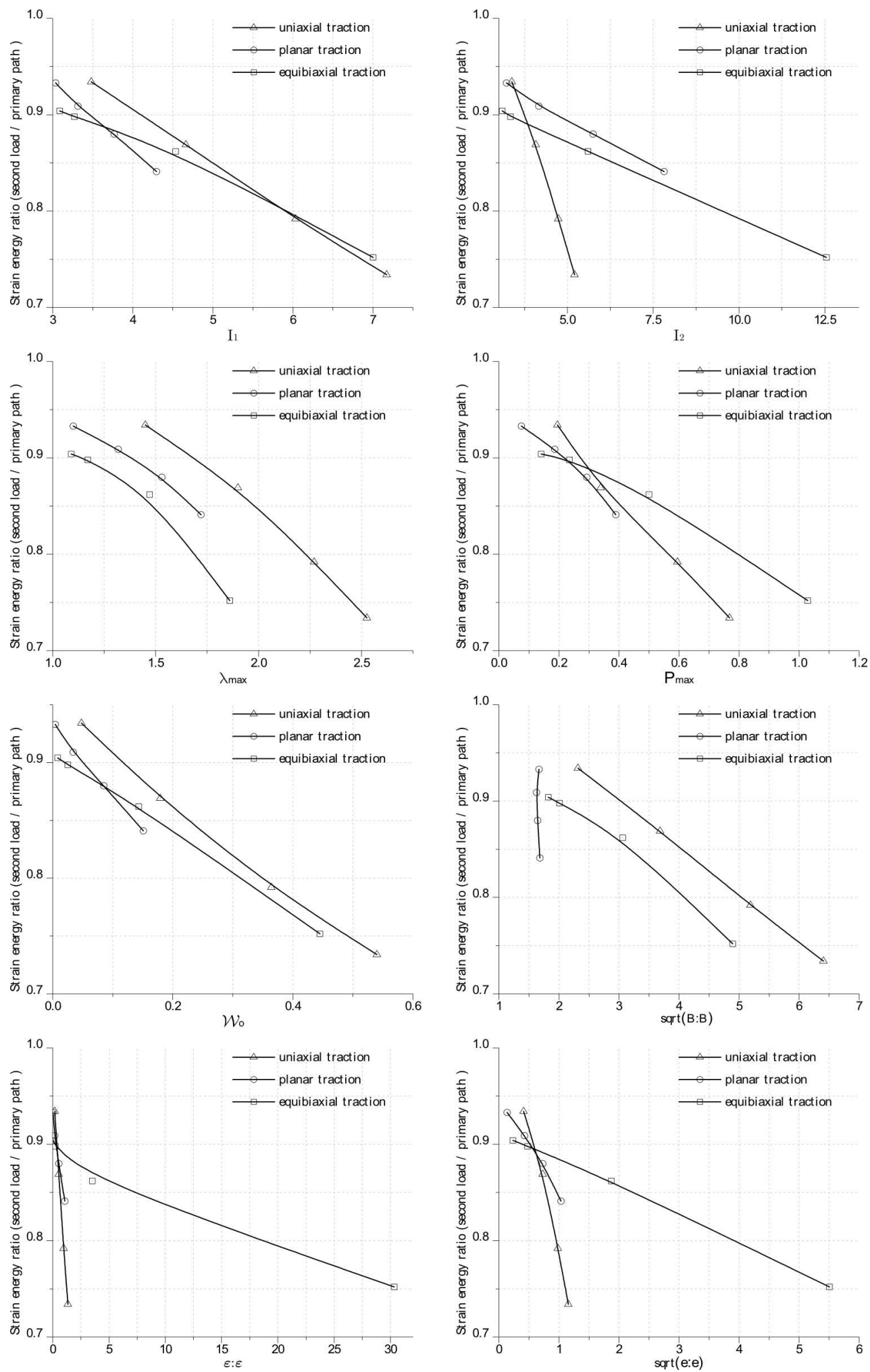

Figure 9: Energy loss between the primary path and the second load for the different mechanical loadings with respect to (a) the first strain invariant $I_{1}$; (b) the second strain invariant $I_{2}$; (c) maximal principal stretch $\lambda_{\max }$; (d) maximal principal stress $P_{\text {max }} ;\left(\right.$ e) strain energy density $\mathcal{W}_{0}$; (f) Left Cauchy-Green strain measure; (g) Piola strain measure; (h) Euler-Almansi strain measure. 

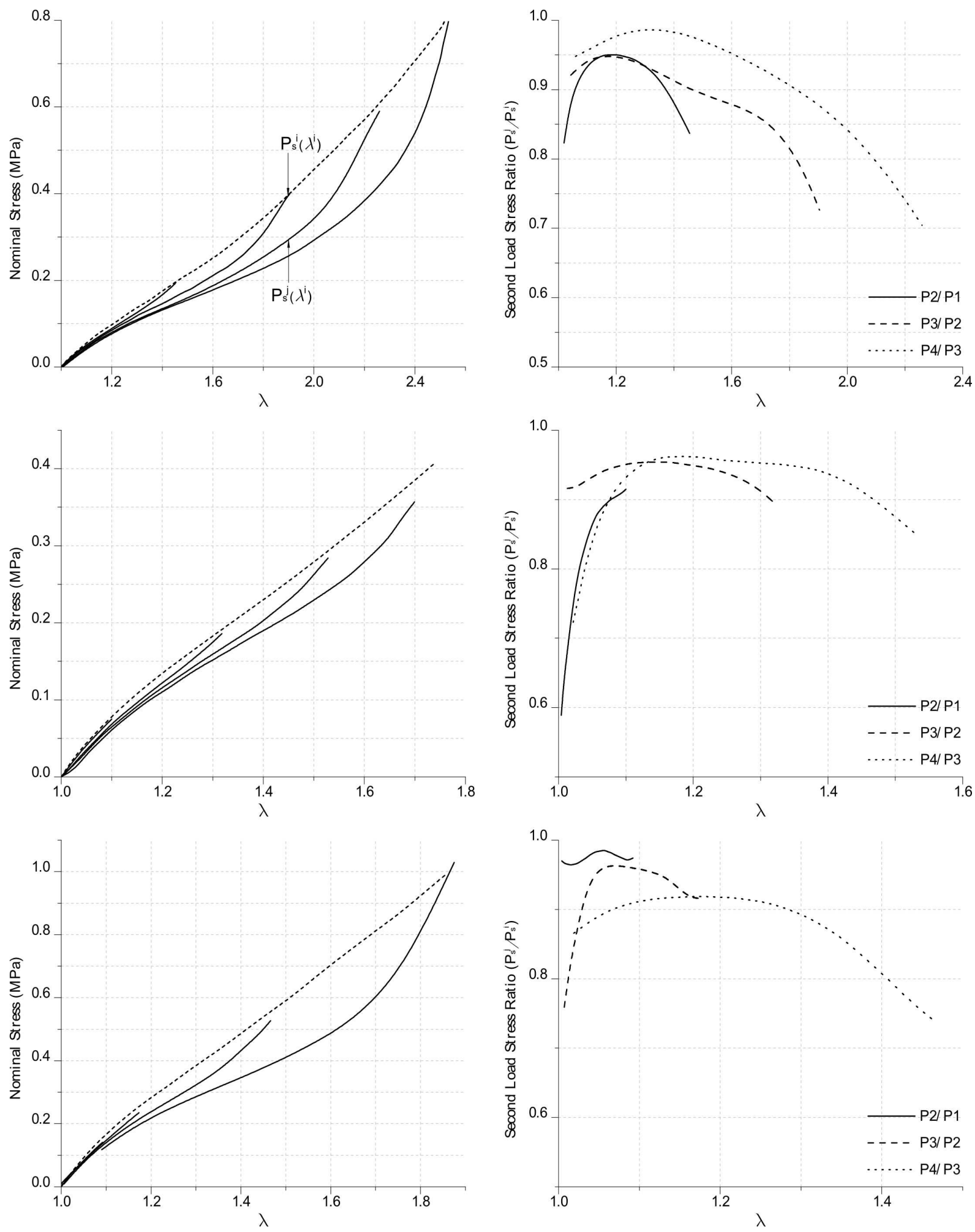

Figure 10: Second loads ratio. Rows correspond, respectively, to uniaxial planar and equibiaxial traction. In the left column dot lines represent the primary path and continuous lines are the second loads. The right column shows the ratio between second loads. 

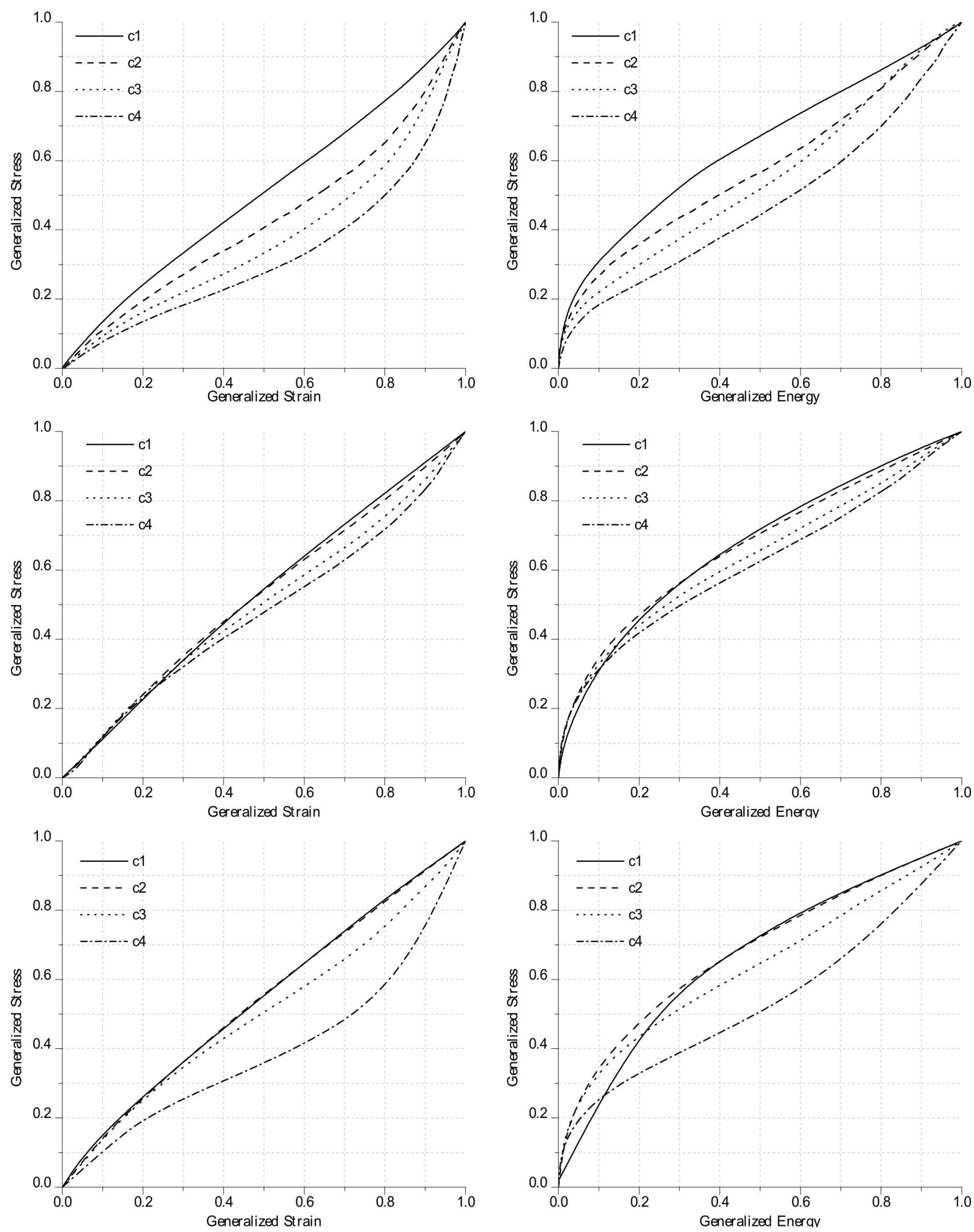

Figure 11: Normalized second stress loading curves with respect the maximum strain (left column) and the normalized energy of deformation (right column). Rows correspond, respectively, to uniaxial planar and equibiaxial traction. 
effect evolution functions to be able to well describe all second loading curves.

\section{Conclusion}

In the present paper, limitation of isotropic models of the Mullins effect have been tackled thanks to experimental tests. In this way, filled silicone rubber has been characterized. The reproducibility and accuracy of measurements were evaluated through several successive tests. Results have shown that our protocol to manufacture the silicone specimens and our experimental methodology work well and provide a precise experimental characterization of softening phenomena. Uniaxial, equibiaxial and planar tensions have been realized, this permits to compare first and second loading curves in different strain modes. Energy losses are compared and analyzed. It clearly appears that the strain energy measure $(\mathcal{W})$ is the best choice and in a lesser the first strain invariant $\left(I_{1}\right)$.

The analysis of experimental data proved that proportionality between second loading curves is not a good way to model the strain-stress curves. There are two main ways to well describe the Mullins effect. The first one, by using a non-constant multiplicative function on second loading curves or by using different non-proportional functions, this respectively corresponds to double-network and physical approaches.

The governing parameters and description function forms have been analyzed under isotropic assumption. The main extension of this study is to, now, take into account the influence of the loading direction of the stress-softening.

\section{Acknowledge}

We would like to thank the French ANR for supporting this work through the project RAAMO ("Robot Anguille Autonome pour Milieux Opaques")

\section{References}

Beatty, M. F. and Krishnaswamy, S. (2000). A theory of stress-softening in incompressible isotropic materials. J. Mech. Phys. Solids, 48, 1931-1965.

Bergstrom, J. S. and Boyce, M. C. (2000). Large strain time-dependant behavior of filled elastomers. Mechanics of materials, 32, 627-644.

Chagnon, G., Verron, E., Gornet, L., Marckmann, G., and Charrier, P. (2004). On the relevance of continuum damage mechanics as applied to the mullinseffect: theory, experiments and numerical implementation. J. Mech. Phys. Solids, 52, $1627-1650$.

Chagnon, G., Verron, E., Marckmann, G., and Gornet, L. (2006). Development of new constitutive equations for mullins effect in rubber usingthe network alteration theory. Int. J. Solids Struct., 43, 6817-6831.

DeSouza Neto, E. A., Djordje Peric, and Owen, D. R. J. (1994). A phenomenological three dimensional rate independent continuum damage modelfor highly filled polymers : formulation and computational aspects. J. Mech. Phys. Solids., 42(10), 1533-1550.

Diani, J., Brieu, M., and Gilormini, P. (2006). Observation and modeling of the anisotropic visco-hyperelastic behaviorof a rubberlike material. Int. J. Solids Struct., 43, 3044-3056.

Diani, J., Fayolle, B., and Gilormini, P. (2009). A review on the mullins effect. Eur. Polym. Journal, 45, 601-612.

Dorfmann, A. and Ogden, R. W. (2004). A constitutive model for the mullins effect with permanent set in particulereinforcedrubber. Int. J. Solids Struct., 41, 1855-1878.

Ehret, A. E. and Itskov, M. (2009). Modeling of anisotropic softening phenomena: Aplication to soft biolgical tissues. Int. J. Plast., 25, 901-919.

Gurtin, M. E. and Francis, E. C. (1981). Simple rate-independent model for damage. J. Spacecraft, 18(3), $285-286$.

Horgan, C. O., Ogden, R. W., and Saccomandi, G. (2004). A theory of stress softening of elastomers based on finite chain extensibility. Proc. R. Soc. London A, 460, 1737-1754.

Johnson, M. A. and Beatty, M. F. (1993). The mullins effect in uniaxial extension and its influence on transversevibration of rubber string. Continuum Mech. Thermodyn., 5, 83-115.

Kazakeviciute-Makovska, R. (2007). Experimentally determined properties of softening functions in pseudo-elasticmodels of the mullins effect. Int. J. Solids Struct., 44, 4145-4157.

Krishnaswamy, S. and Beatty, M. F. (2000). The mullins effet in compressible solids. Int. J. Eng. Sci., 38, 1397-1414.

Laiarinandrasana, L., Piques, R., and Robisson, A. (2003). Visco-hyperelastic model with internal state variable coupled with discontinuousdamage concept under total lagrangian formulation. Int. J. Plast., 19, 977-1000. 
Laraba-Abbes, F., Ienny, P., and Piques, R. (2003). A new taylor-made methodology for the mechanical behaviour analysisof rubber like materials: II. application of the hyperelastic behaviourcharacterization of a carbon-black filled natural rubber vulcanizate. Polymer, 44, 821-840.

Lion, A. (1996). A constitutive model for carbon black filled rubber: Experimental investigationsand mathematical representation. Continuum Mech. Thermodyn., 8, 153-169.

Marckmann, G., Verron, E., Gornet, L., Chagnon, G., and Fort, P. C. P. (2002). A theory of network alteration for the mullins effect. J. Mech. Phys. Solids., 50, 2011-2028.

Meunier, L., Chagnon, G., Favier, D., Orgéas, L., and Vacher, P. (2008). Mechanical experimental characterisation and numerical modelling of an unfilledsilicone rubber. Polym. Test., 27, 765-777.

Miehe, C. (1995). Discontinuous and continuous damage evolution in ogden type large strainelastic materials. Eur. J. Mech., A/Solids, 14(5), 697-720.

Miehe, C. and Göktepe, S. (2005). A micro-macro approach to rubber-like materials. part iii: The micro-sphere model anisotropic mullins-type damage. J. Mech. Phys. Solids, 53, 2259-2283.

Miehe, C. and Keck, J. (2000). Superimposed finite elastic-viscoelastic-plastoelastic stress response withdamage in filled rubbery polymers. experiments, modelling and algorithmicimplementation. J. Mech. Phys. Solids, 48, 323-365.

Mullins, L. (1969). Softening of rubber by deformation. Rubber Chem. Technol., 42, 339-362.

Ogden, R. W. and Roxburgh, D. G. (1999). A pseudo-elastic model for the mullins effect in filled rubber. Proc. R. Soc. Lond. A, 455, 2861-2877.

Qi, H. J. and Boyce, M. C. (2004). Constitutive model for stretch-induced softening of the stress-stretch behaviorof elastomeric materials. J. Mech. Phys. Solids, 52, 2187-2205.

Sasso, M., Palmieri, G., Chiappini, G., and Amodio, D. (2008). Characterization of hyperelastic rubber-like materials by biaxial and uniaxialstretching tests based on optical methods. Polym. Test., 27, 995-1004.

Septanika, E. G. and Ernst, L. J. (1998). Application of the network alteration theory for modeling the time dependent constitutive behavior of rubber. Part I - general theory. Mechanics of materials, 30, 253-263.

Simo, J. C. (1987). On a fully three-dimensional finite-strain viscoelastic damage model : formulationand computational aspects. Comp. Meth. Appl. Mech. Engng, 60, 153-173.

Sutton, M. A. (2008). Digital image correlation for shape and deformation measurements. Springer Handbook of Experimental Solid Mechanics - PartC, pages 565-600.

Zuñiga, A. E. (2005). A phenomenological energy-based model to characterize stress-softening effectin elastomers. Polymer, 46, 3496-3506.

Zuñiga, A. E. and Beatty, M. F. (2002). A new phenomenological model for stress-softening in elastomers. Z. Angew. Math. Phys., 53, 794-814. 